\title{
Long non-coding RNA DLX6-AS1 silencing inhibits malignant phenotypes of gastric cancer cells
}

\author{
XIAODAN FU ${ }^{1}$, YIFU TIAN $^{1}$, WEILU KUANG $^{2}$, SAILAN WEN $^{3}$ and WEI GUO ${ }^{4}$ \\ Departments of ${ }^{1}$ Pathology and ${ }^{2}$ Oncology, Xiangya Hospital, Central South University, Changsha, Hunan 410005; \\ ${ }^{3}$ Department of Pathology, Second Xiangya Hospital, Central South University, Changsha, Hunan 410011; \\ ${ }^{4}$ Department of Pathology, Hunan Provincial People's Hospital, The First Affiliated Hospital of \\ Hunan Normal University, Changsha, Hunan 410002, P.R. China
}

Received August 16, 2018; Accepted April 1, 2019

DOI: $10.3892 /$ etm.2019.7521

\begin{abstract}
It has been revealed that long non-coding RNAs (lncRNAs) serve a key role in various malignancies, including gastric cancer (GC). In the present study, the expression and function of lncRNA distal-less homeobox 6 antisense 1 (DLX6-AS1) in GC was investigated. The data revealed that the expression of DLX6-AS1 was significantly upregulated in GC tissues compared with adjacent paired noncancerous tissues. Furthermore, the expression of DLX6-AS1 was higher in advanced GC tissue samples (III/IV) compared with the expression in early-stage samples (I/II). Furthermore, the current study demonstrated that a high expression of DLX6-AS1 was significantly associated with advanced clinical stage, lymph node metastasis and distant metastasis. Compared with patients with a low DLX6-AS1 expression, DLX6-AS1 expression in patients with GC was associated with decreased survival. In vitro experimental data indicated that DLX6-AS1 was upregulated in GC cell lines and that the inhibition of DLX6-AS1 markedly reduced GC cell proliferation, colony formation, cell cycle progression, migration and invasion. Further investigation revealed that knockdown of DLX6-AS1 inhibited EMT in GC cells. In summary, the present study demonstrated that IncRNA DLX6-AS1 was upregulated and serves an oncogenic role in GC, indicating that DLX6-AS1 may be a novel therapeutic target for GC treatment.
\end{abstract}

Correspondence to: Dr Yifu Tian, Department of Pathology, Xiangya Hospital, Central South University, 87 Xiangya Road, Changsha, Hunan 410005, P.R. China

E-mail: tave1231@163.com

Key words: long non-coding RNA, distal-less homeobox 6 antisense 1 , gastric cancer, prognosis

\section{Introduction}

Gastric cancer (GC) is the fourth most common cancer worldwide $(1,2)$. GC originates from the mucosal epithelium of the stomach and causes a significant number of mortalities worldwide $(1,2)$. GC is difficult to diagnose at an early stage and thus the majority of patients with GC present with advanced disease or tumor metastasis, making the disease difficult to treat (3). In recent decades, although great progress has been made in GC treatment, including surgical resection combined with chemotherapy, the survival of patients with advanced GC has not improved (1-3). It is therefore necessary to assess the molecular mechanisms underlying GC development and progression, which may help identify novel and promising therapeutic targets for this disease.

The human genome produces coding and non-coding RNAs, the latter of which is the predominant RNA species $(4,5)$. Long non-coding RNAs (lncRNAs), a class of non-coding RNAs that are comprised of $>200$ nucleotides, often exhibit spatial and temporal-specific expression patterns $(4,5)$. It has been widely reported that lncRNAs serve important roles in regulating a variety of cellular biological processes, including cell proliferation, apoptosis, migration, invasion and tumourigenesis (6-9). Furthermore, a large number of lncRNAs are dysregulated during the development or progression of certain types of human cancer, including GC (10-14). For instance, the lncRNA X-inactive specific transcript (XIST) is significantly upregulated in GC cell lines and the knockdown of XIST inhibits the growth of GC cells (15). Small Nucleolar RNA Host Gene 12 (SNHG12), which is associated with tumor size and metastasis, is overexpressed in GC (16). The inhibition of SNHG12 reduces GC cell growth, colony formation, proliferation and invasion (16).

Distal-less homeobox 6 antisense 1 (DLX6-AS1), a lncRNA localized at the 7q21.3 chromosomal region, has been revealed to be frequently upregulated, serving an oncogenic role in several types of common cancer, including lung adenocarcinoma (17), renal cell carcinoma (18) and hepatocellular carcinoma (19). For instance, DLX6-AS1 levels are significantly higher in lung adenocarcinoma tissues than in adjacent healthy lung tissues and its overexpression is closely associated with poor histological differentiation and advanced 
TNM stage (17). However, to the best of our knowledge, the expression and function of DLX6-AS1 in GC has not been previously studied.

The present study therefore aimed to assess DLX6-AS1 expression in GC tissues and cell lines and to examine the association between DLX6-AS1 expression and GC clinical characteristics. Furthermore, the function of DLX6-AS1 in cell proliferation, apoptosis, migration and invasion was assessed in GC in vitro.

\section{Materials and methods}

Clinical tissue samples. The present study was approved by the Ethics Committee of Xiangya Hospital (Changsha, China). A total of 62 GC tissue and matched adjacent healthy tissue samples (3 $\mathrm{cm}$ from the tumor edge) were collected from 62 primary patients with GC admitted to Xiangya Hospital (Changsha, China) between March 2015 and June 2017 (Table I). Written informed consent had been previously obtained. None of the patients involved in the current study underwent chemotherapy or radiotherapy prior to surgery. After surgical resection, tissues were frozen using liquid nitrogen and stored at $-80^{\circ} \mathrm{C}$ until further use.

Cell culture. Normal human gastric mucosa epithelial GES-1 cells and GC cell lines (including HGC27, BGC823, SGC7901 and AGS cells) were purchased from the Cell Bank of Chinese Academy of Sciences. Cell lines were cultured in DMEM (Thermo Fisher Scientific, Inc.) supplemented with $10 \%$ FBS (Thermo Fisher Scientific Inc.) at $37^{\circ} \mathrm{C}$ in a humidified atmosphere with $5 \% \mathrm{CO}_{2}$.

Cell transfection. AGS and BGC823 cells ( $1 \times 10^{6}$ cells/well) were seeded in six-well plates and cultured at $37^{\circ} \mathrm{C}$ to $\sim 80 \%$ confluence. Cells were subsequently transfected with 100 nM DLX6-AS1 small interfering RNA (siRNA; siDLX6-AS1) or $100 \mathrm{nM}$ negative control siRNA (siNC; both Shanghai GenePharma Co., Ltd.) using Lipofectamine ${ }^{\circledR} 2000$ (Thermo Fisher Scientific, Inc.), according to the manufacturer's protocol. Following 48-h transfection, cells were used in further experimentation.

Reverse transcription-quantitative (RT-q) PCR. Total RNA was extracted from tissues or cell lines using TRIzol ${ }^{\circledR}$ reagent (Thermo Fisher Scientific, Inc.). Total RNA $(1 \mu \mathrm{g})$ was reverse transcribed into cDNA using the High Capacity cDNA Reverse Transcription kit (Thermo Fisher Scientific, Inc.), according to manufacturer's protocol. qPCR was performed using SYBR Green Reverse Transcription PCR Master mix (Takara Biotechnology Co., Ltd.) on an Applied Biosystems 7300 plus reverse transcription PCR system (Thermo Fisher Scientific, Inc.). The following primer pairs were used for the qPCR: DLX6-AS1 forward, 5'-AGTTTCTCTCTAGATTGCCTT-3' and reverse, 5'-ATTGACATGTTAGTGCCCTT-3'; GAPDH forward, 5'-CTGGGCTACACTGAGCACC-3' and reverse, 5'-AAGTGGTCGTTGAGGGCAATG-3'. The following thermocycling conditions were used for the qPCR: Initial denaturation at $95^{\circ} \mathrm{C}$ for $30 \mathrm{sec} ; 40$ cycles of $95^{\circ} \mathrm{C}$ for $5 \mathrm{sec}$ and $60^{\circ} \mathrm{C}$ for $30 \mathrm{sec}$. Expression levels were quantified using the $2^{-\triangle \Delta C q}$ method and GAPDH was used as an internal reference gene (20).
Cell counting kit-8 (CCK-8) assay. AGS and BGC823 cells $\left(5 \times 10^{3}\right.$ cells/well $)$ were plated into 96 -well plates and incubated at $37^{\circ} \mathrm{C}$ for $0,24,48$ or $72 \mathrm{~h}$. CCK-8 reagent (10 $\mu \mathrm{l}$; Beyotime Institute of Biotechnology) was then added to each well. After incubation at $37^{\circ} \mathrm{C}$ for $2 \mathrm{~h}$, the optical density absorbance at $450 \mathrm{~nm}$ was measured using a microplate reader.

Colony formation assay. Transfected AGS and BGC823 cells $\left(1.5 \times 10^{2}\right.$ cells/well) were seeded into six-well plates and cultured in DMEM with $10 \% \mathrm{FBS}$ at $37^{\circ} \mathrm{C}$ for 10 days. Cells were then washed with DPBS (Thermo Fisher Scientific, Inc.) and stained with $0.1 \%$ crystal violet for $5 \mathrm{~min}$ at room temperature. The number of colonies consisting of $>50$ cells were counted under a light microscope (magnification, $\mathrm{x} 40$; Olympus Corporation).

Flow cytometry for cell cycle analysis. Transfected AGS and BGC 823 cells were washed with PBS and fixed in $75 \%$ ethanol overnight at $4^{\circ} \mathrm{C}$. Cells were subsequently washed three times with PBS and stained with $500 \mu 1$ eBioscience ${ }^{\mathrm{TM}}$ propidium iodide staining solution (cat. no. 00-6990-50; Thermo Fisher Scientific, Inc.) at room temperature for $15 \mathrm{~min}$. Cell cycle distribution was examined using a FACScan flow cytometer (BD Biosciences) and BD Accuri C6 system software (version 1.0; BD Biosciences).

Wound healing assay. Transfected AGS and BGC823 cells (1x10 ${ }^{6}$ cells/well) were seeded into six-well plates and cultured in DMEM with $10 \% \mathrm{FBS}$ at $37^{\circ} \mathrm{C}$ at $\sim 24 \mathrm{~h}$ to $\sim 90 \%$ confluence. Wounds were created using a $200 \mu \mathrm{l}$ pipette tip. Wound healing within the scrape line was observed and imaged at 0 and $24 \mathrm{~h}$ using a light microscope (magnification, $\mathrm{x} 400$; Olympus Corporation).

Transwell cell invasion assay. A transwell assay was performed using a 24 -well transwell chamber $(8 \mathrm{~mm}$ pore size; Corning, Inc.) pre-coated with Matrigel Basement Membrane Matrix (BD Biosciences). Transfected cells $\left(3 \times 10^{3}\right.$ cells/well) in $300 \mu \mathrm{l}$ DMEM without serum were added to the upper chamber, while $500 \mu \mathrm{l}$ DMEM with $10 \%$ FBS was added to the lower chamber. Cells were then incubated at $37^{\circ} \mathrm{C}$ for $24 \mathrm{~h}$. Cells inside the insert were removed using a cotton-tipped swab. Cells that had invaded were fixed with $4 \%$ paraformaldehyde (Sigma-Aldrich; Merck KGaA) for $30 \mathrm{~min}$ at room temperature. Fixed cells were subsequently stained with $0.5 \%$ crystal violet (Beyotime Institute of Biotechnology) for $10 \mathrm{~min}$ at room temperature. Invading cells were observed under a light microscope (magnification, x400; Olympus Corporation).

Western blot analysis. Transfected AGS and BGC823 cells were lysed using RIPA buffer (Beyotime Institute of Biotechnology). Protein concentration was determined using the Pierce BCA Protein Assay kit (Thermo Fisher Scientific, Inc.), according to the manufacturer's protocol. Protein samples $(50 \mu \mathrm{g})$ were separated via SDS-PAGE on a $10 \%$ gel. The separated proteins were transferred onto nitrocellulose membranes (EMD Millipore) and blocked with 5\% non-fat dry milk overnight at $4^{\circ} \mathrm{C}$. The membranes were incubated with primary antibodies against E-cadherin (1:500; ab133597), N-cadherin (1:500; ab245117), Vimentin (1:200; ab16700) and GAPDH (1:500; 
A

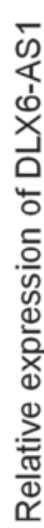
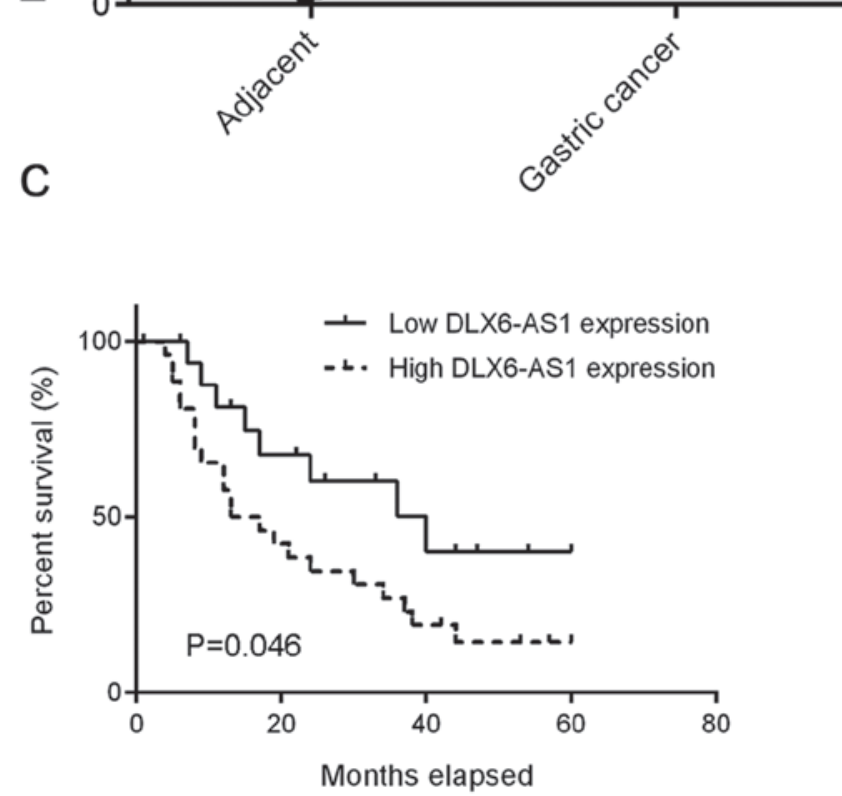

B

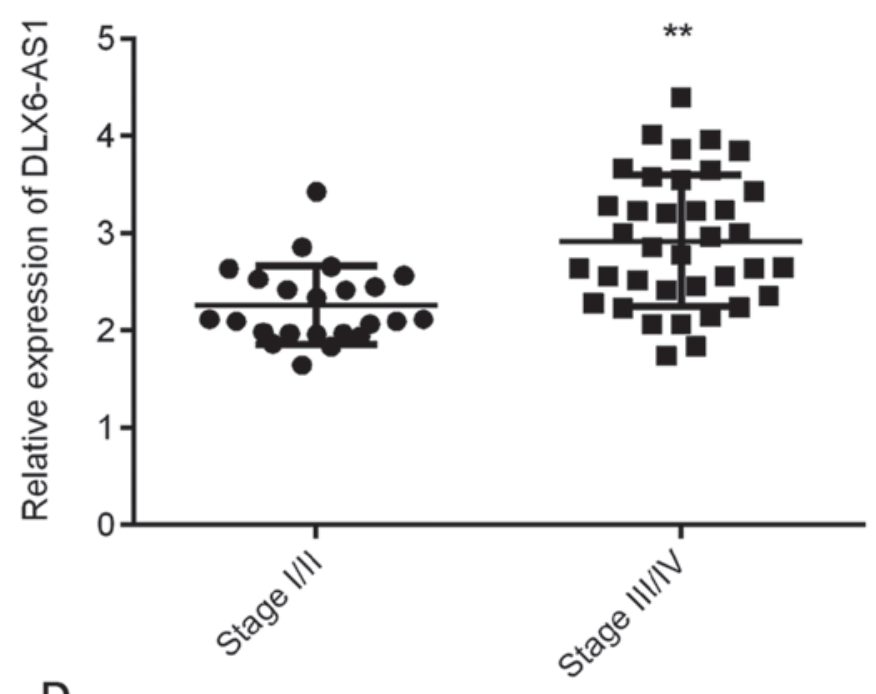

D

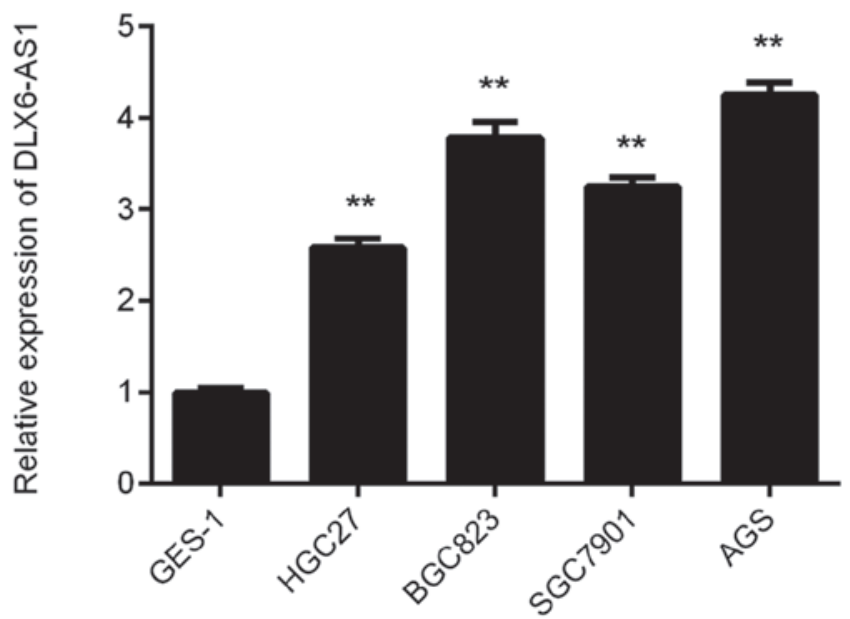

Figure 1. Upregulation of DLX6-AS1 in gastric cancer. (A) DLX6-AS1 expression is significantly increased in gastric cancer tissues compared with adjacent normal tissues, as determined via reverse transcription-quantitative PCR. ${ }^{* *} \mathrm{P}<0.01$ vs. Adjacent. (B) The expression of DLX6-AS1 was higher in advanced gastric cancer tissue samples (III/IV) compared with early-stage samples (I/II). ${ }^{* *} \mathrm{P}<0.01$ vs. Stage I/II. (C) Patients with gastric cancer and a high DLX6-AS1 expression exhibited shorter survival time than those with a lower expression of DLX6-AS1. (D) DLX6-AS1 expression was significantly higher in gastric cancer cell lines than in the GES-1 cell line. ${ }^{* *} \mathrm{P}<0.01$ vs. GES-1. DLX6-AS1, distal-less homeobox 6 antisense 1.

ab9485; all Abcam) for $3 \mathrm{~h}$ at room temperature. Following primary incubation, membranes were incubated with horseradish peroxidase-conjugated goat anti-rabbit second antibody (1:5,000; ab6721; Abcam) for $1 \mathrm{~h}$ at room temperature. Protein bands were visualized using the SuperSignal West Femto Maximum Sensitivity substrate (Thermo Fisher Scientific, Inc.). Protein expression was quantified using ImageJ software (version 1.46; National Institutes of Health).

Statistical analysis. Data presented as the mean \pm standard deviation. SPSS version 22.0 for windows (IBM Corp.) was used for all analyses. A Student's t-test was used for analysing the differences between two groups. One-way ANOVA followed by a Tukey's post-hoc test was used for the comparison of $>2$ groups. A Chi-square test was used to analyse the association between DLX6-AS1 expression and the clinicopathological characteristics of patients with GC. Kaplan-Meier survival curves were analysed with a log-rank test. $\mathrm{P}<0.05$ was considered to indicate a statistically significant difference.

\section{Results}

Upregulation of DLX6-AS1 in GC. To assess the biological function of DLX6-AS1 in GC, RT-qPCR analysis was performed to determine its expression in a total of 62 primary GC tissues and their matched adjacent normal tissues. The data revealed that DLX6-AS1 expression was significantly increased in GC tissues compared with adjacent normal tissues (Fig. 1A). The expression of DLX6-AS1 was also observed to be higher in advanced GC tissue samples (III/IV) compared with early-stage samples (I/II; Fig. 1B), indicating that the upregulation of DLX6-AS1 was associated with GC progression. To further clarify whether DLX6-AS1 expression was associated with the clinicopathological characteristics of patients with GC, patients were divided into DLX6-AS1 low expression and high expression groups using the median level of DLX6-AS1 as a cut-off (2.67). As indicated in Table I, high DLX6-AS1 expression was not associated with sex, age, tumor size or differentiation, but was significantly associated 
Table I. Association between DLX6-AS1 expression and the clinicopathological characteristics of patients with gastric cancer.

\begin{tabular}{|c|c|c|c|c|}
\hline \multirow[b]{2}{*}{ Variables } & \multirow[b]{2}{*}{ Cases $(n=62)$} & \multicolumn{2}{|c|}{ DLX6-AS1 expression } & \multirow[b]{2}{*}{ P-value } \\
\hline & & Low levels $(\mathrm{n}=33)$ & High levels $(n=29)$ & \\
\hline Age (years) & & & & 0.794 \\
\hline$\leq 7$ & 23 & 13 & 10 & \\
\hline$>60$ & 39 & 20 & 19 & \\
\hline Sex & & & & 0.302 \\
\hline Male & 37 & 22 & 15 & \\
\hline Female & 25 & 11 & 14 & \\
\hline Tumor size $(\mathrm{cm})$ & & & & 0.322 \\
\hline$\leq 32$ & 30 & 18 & 12 & \\
\hline$>5 \mathrm{~cm}$ & 32 & 15 & 17 & \\
\hline Differentiation & & & & 0.068 \\
\hline Well and moderately & 38 & 24 & 14 & \\
\hline Poor & 24 & 9 & 15 & \\
\hline Node metastasis & & & & $0.024^{\mathrm{a}}$ \\
\hline Present & 44 & 19 & 25 & \\
\hline Absent & 18 & 14 & 4 & \\
\hline Distant metastasis & & & & $0.018^{\mathrm{a}}$ \\
\hline Present & 16 & 4 & 12 & \\
\hline Absent & 46 & 29 & 17 & \\
\hline Clinical stage & & & & $0.018^{\mathrm{a}}$ \\
\hline I-II & 23 & 17 & 6 & \\
\hline III-IV & 39 & 16 & 23 & \\
\hline
\end{tabular}

${ }^{\mathrm{a}} \mathrm{P}<0.05$. DLX6-AS1, distal-less homeobox 6 antisense 1 .

with metastasis and advanced TNM stage. The results indicate that patients with GC and a high DLX6-AS1 expression exhibit a lower percentage survival than those with a low DLX6-AS1 expression (Fig. 1C). These results indicate that DLX6-AS1 expression may be used as a predictive marker for GC prognosis and as such, further study should be conducted to explore the function of DLX6-AS1 in GC. The expression of DLX6-AS1 was subsequently examined in a normal human gastric mucosa epithelial cell line, GES-1 and several GC cell lines, including HGC27, BGC823, SGC7901 and AGS. As presented in Fig. 1D, DLX6-AS1 expression was significantly higher in GC cell lines compared with GES-1 cells. AGS and BGC823 cell lines were subsequently selected to perform in vitro experiments, as they exhibited the highest levels of DLX6-AS1 expression.

Inhibition of DLX6-AS1 suppresses GC cell proliferation, colony formation and cell cycle progression. To examine the function of DLX6-AS1 in GC in vitro, AGS and BGC823 cells were transfected with DLX6-AS1 siRNA to downregulate its expression. At $48 \mathrm{~h}$ after transfection, RT-qPCR was performed to assess DLX6-AS1 expression. As presented in Fig. 2A, the expression of DLX6-AS1 was significantly reduced in the siDLX6-AS1 group compared with the siNC group. A CCK-8 assay was then performed in order to assess cell proliferation. The results revealed that the proliferation of
AGS and BGC823 cells was significantly inhibited at 48 and $72 \mathrm{~h}$ in the siDLX6-AS1 group compared with the siNC group (Fig. 2B and C). These results indicate that DLX6-AS1 may serve a growth-promoting role in GC. To confirm this, a colony formation assay was performed, the results of which indicated that the colony formation capacity of cells was significantly reduced in the siDLX6-AS1 group compared with the siNC group (Fig. 2D). Cell cycle regulation serves a key role in cell proliferation (21). Therefore, flow cytometry was performed to assess the function of DLX6-AS1 in the regulation of cell cycle progression. As presented in Fig. 2E and F, the knockdown of DLX6-AS1 caused significant G1 and G2-M stage arrest compared with siNC cells in AGS and BGC823 cells. The aforementioned data indicates that DLX6-AS1 may promote cell proliferation, colony formation and cell cycle progression in GC.

Silencing of DLX6-AS1 inhibits GC cell migration, invasion and epithelial-mesenchymal transition (EMT). Tumor cell migration and invasion are essential for tumor metastasis (22). Wound healing and transwell assays were performed to assess the function of DLX6-AS1 in GC cell migration and invasion. As presented in Fig. 3A and B, wound healing assay data revealed that compared with cells in the siNC group, the migratory capacity of DLX6-AS1 inhibited AGS and BGC823 cells was significantly reduced. Similarly, fewer invasive cells were 

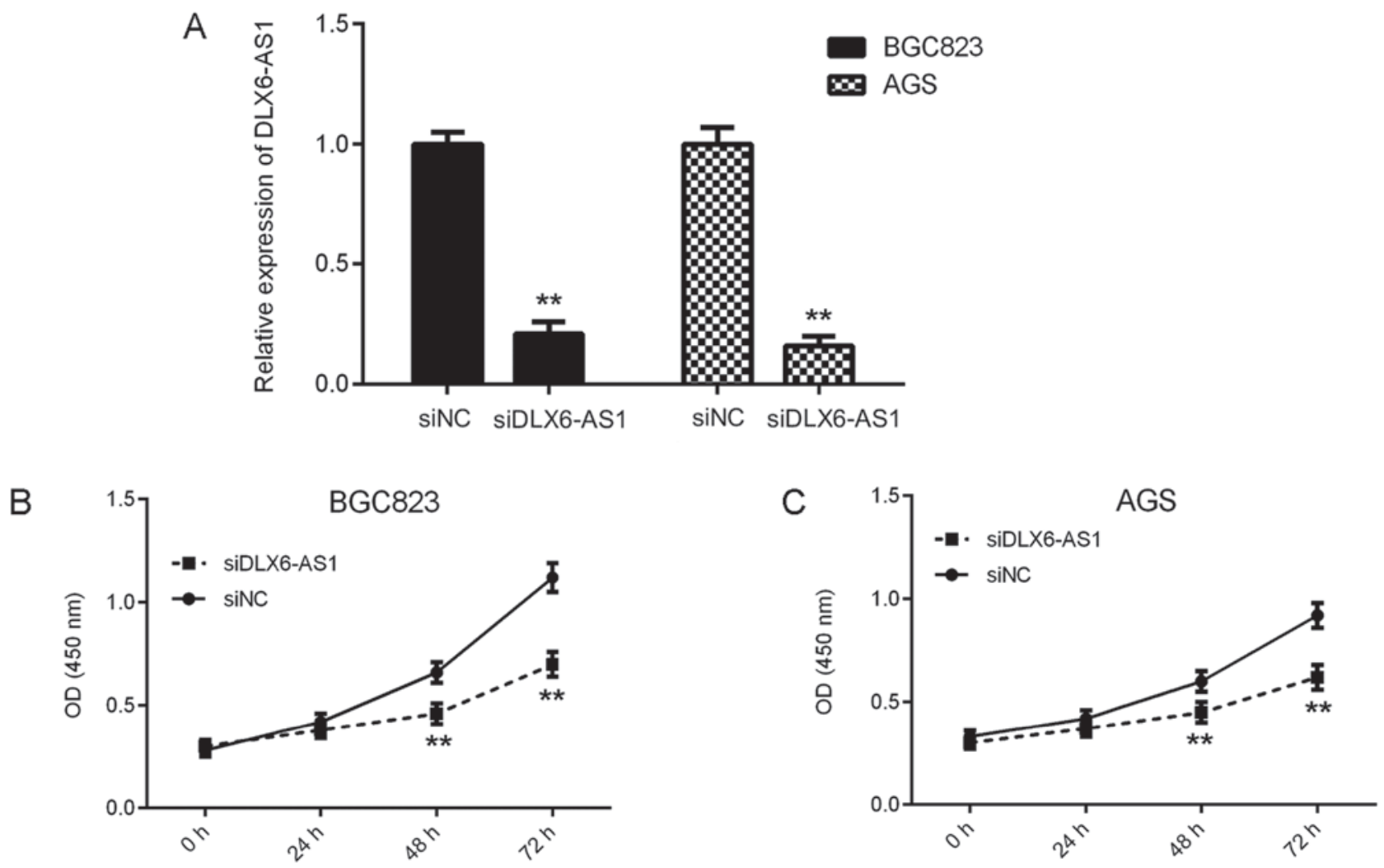

D BGC823

\section{AGS}
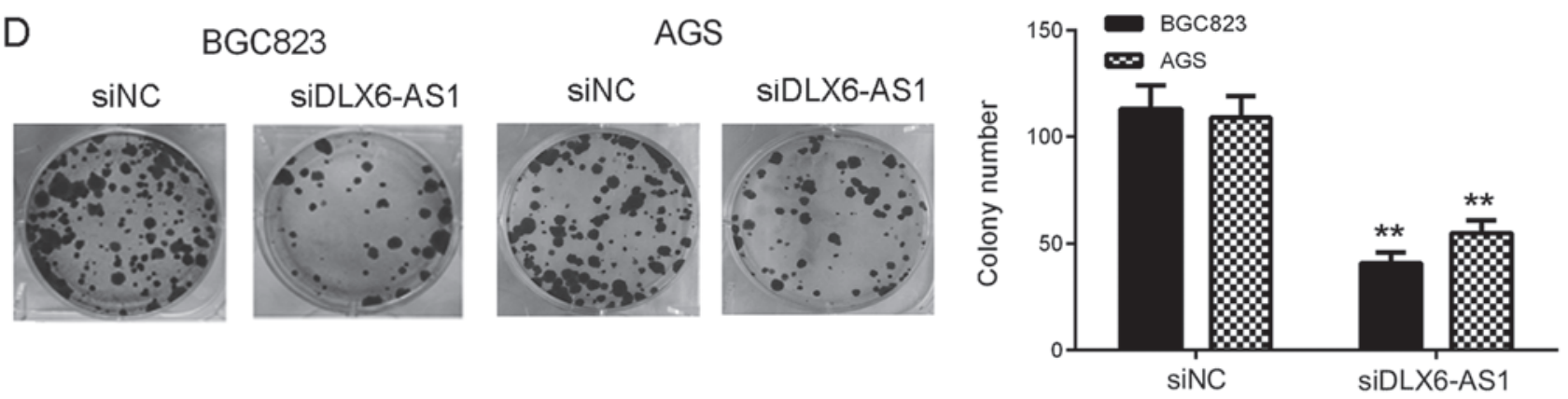

$\mathrm{E}$

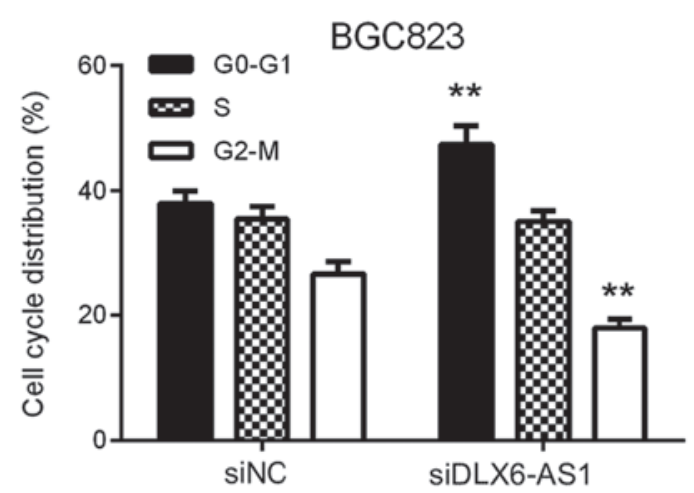

$\mathrm{F}$

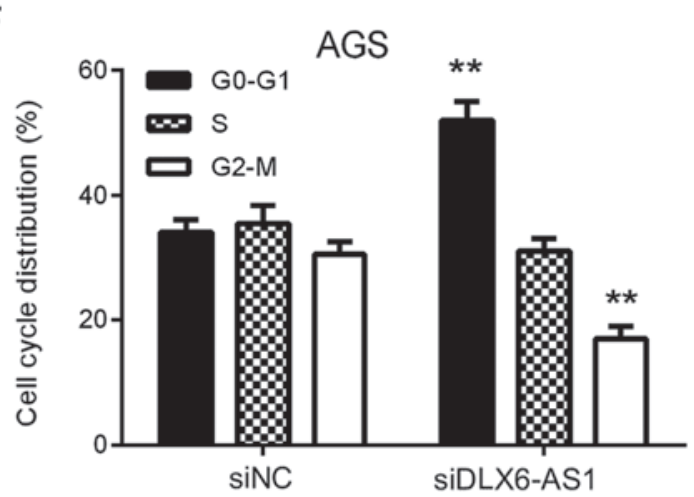

Figure 2. Inhibition of DLX6-AS1 suppressed GC cell proliferation, colony formation and cell cycle progression. AGS and BGC823 cells were transfected with DLX6-AS1 siRNA or NC siRNA. After transfection, (A) the expression of DLX6-AS1 was examined. A cell counting kit-8 assay was performed to measure (B) cell proliferation and (C) AGS. (D) Colony formation and (E) BGC823 and (F) AGS cell cycle distributions were also examined. ** P $<0.01$ vs. siNC. DLX6-AS1, Distal-less homeobox 6 antisense 1; GC, gastric cancer; siRNA, small interfering RNA; NC, negative control; OD, optical density.

observed in the siDLX6-AS1 group than in the siNC group, indicating that DLX6-AS1 silencing inhibits GC cell invasion (Fig. 3C and D). These data indicate that DLX6-AS1 serves a promoting role in the migration and invasion of GC cells, which may contribute to GC metastasis.
As EMT serves a key role during tumor cell migration and invasion (23), the expression of EMT markers in AGS and BGC823 cells were assessed with or without DLX6-AS1 inhibition. The results of western blot analysis demonstrated that DLX6-AS1 silencing expression caused the significant 
A

BGC823

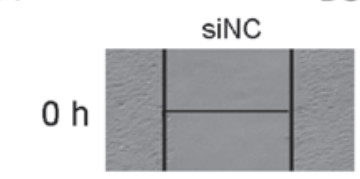

siDLX6-AS1

$24 \mathrm{~h}$
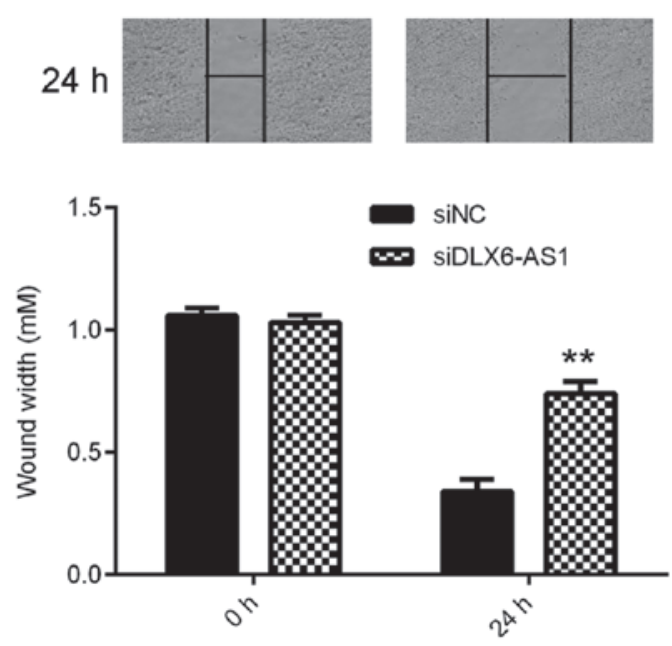

C BGC823
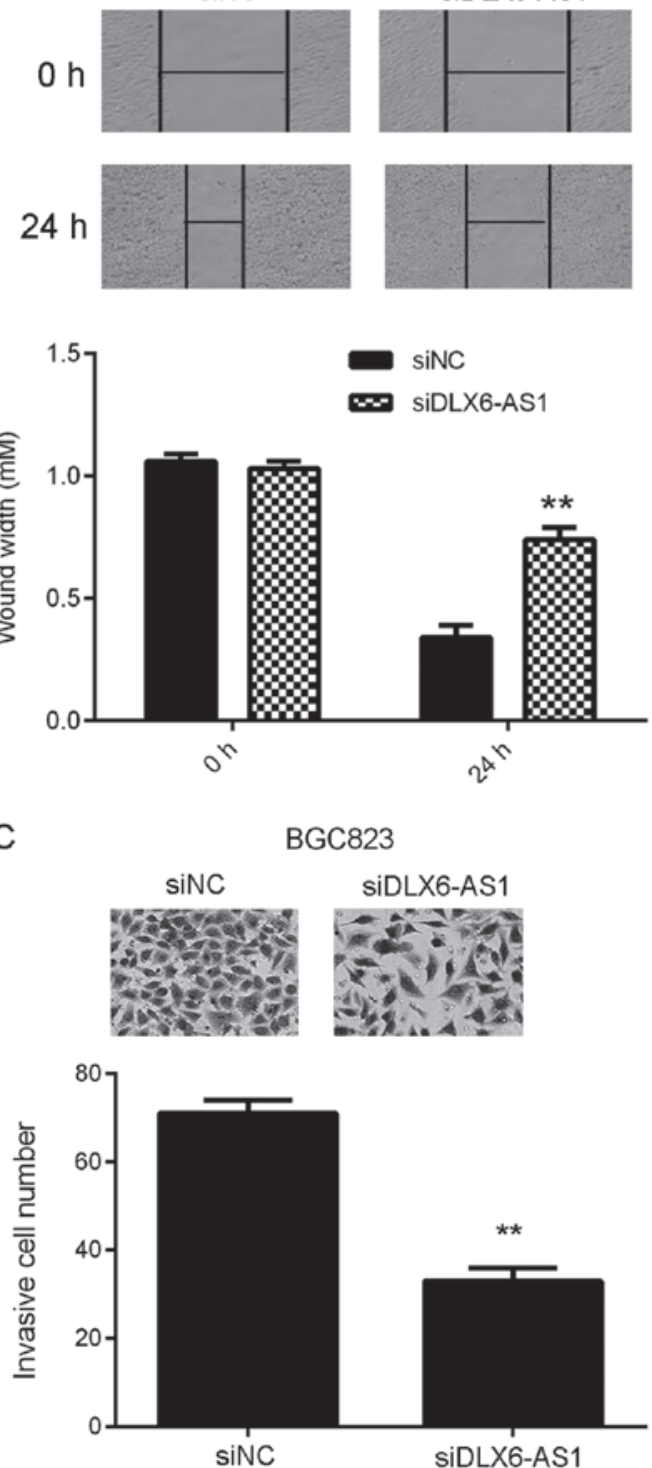

B

$\mathrm{Oh}$

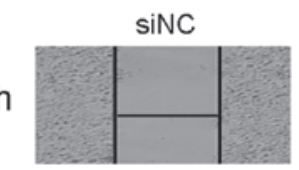

AGS

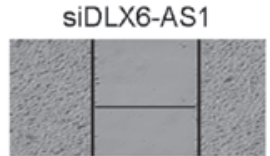

$24 \mathrm{~h}$
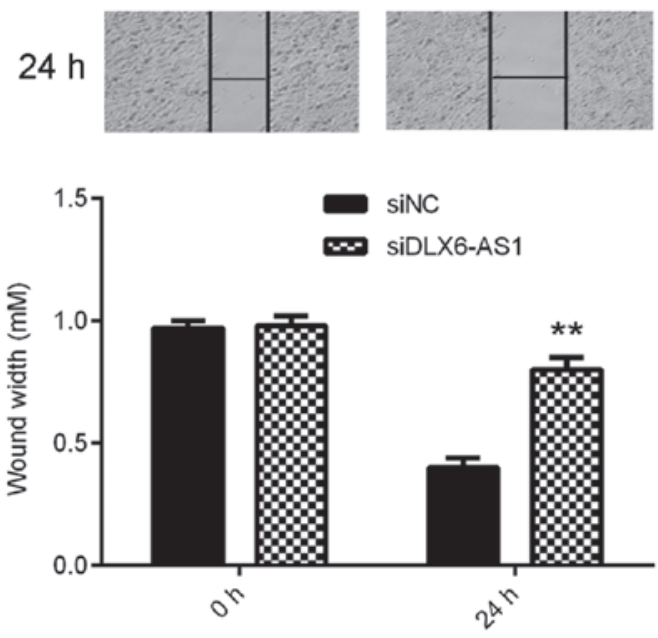

D
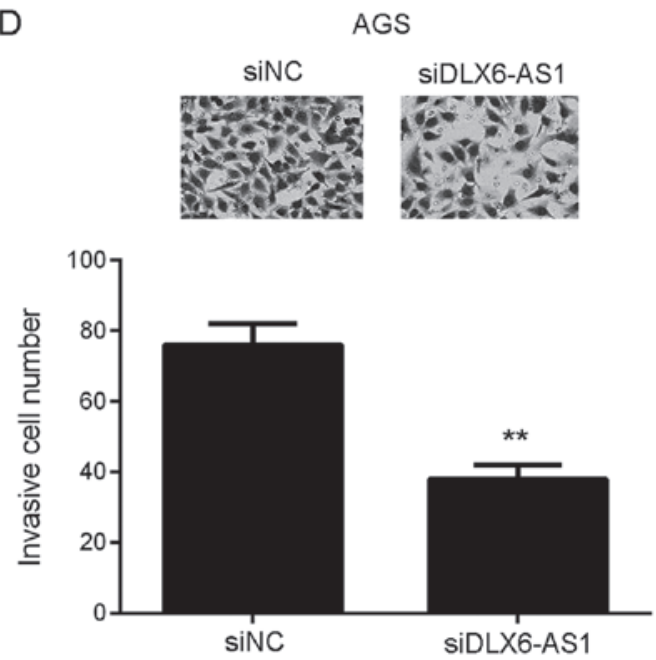

Figure 3. Silencing of DLX6-AS1 inhibited gastric cancer cell migration and invasion. (A) BGC823 and (B) AGS cells were transfected with DLX6-AS1 siRNA or NC siRNA and a wound healing assay was performed to assess cell migration. A transwell assay was used to examine (C) BGC823 and (D) AGS cell invasion. ${ }^{* *} \mathrm{P}<0.01$ vs. siNC. DLX6-AS1, distal-less homeobox 6 antisense 1; siRNA, small interfering RNA; NC, negative control.

upregulation of E-cadherin (an epithelial marker) and the marked downregulation of $\mathrm{N}$-cadherin and Vimentin (mesenchymal markers) in AGS and BGC823 cells compared with the cells in the siNC group (Fig. 4). The results indicate that the knockdown of DLX6-AS1 suppresses GC cell migration and invasion by inhibiting EMT.

\section{Discussion}

Although non-protein coding RNAs occupy $>90 \%$ of the human genome's transcriptional output (7), the molecular mechanisms of lncRNAs underlying cancer development and progression remain largely unknown. The current study revealed that the expression of DLX6-AS1 was significantly increased in GC tissues and cell lines and that its upregulation was associated with advanced clinical stage, lymph node metastasis, distant metastasis and poor prognosis in patients with GC. The knockdown of DLX6-AS1 inhibited GC cell proliferation, colony formation, cell cycle progression, migration, invasion and EMT in vitro.

In recent years, a large number of lncRNAs have been identified as key regulators during cancer development and progression $(24,25)$. The poor prognosis of patients with advanced GC is primarily thought to be due to rapid tumor metastasis (26). This therefore reveals that furthering understanding into the underlying mechanisms of GC may be beneficial for the development of novel and effective therapies $(27,28)$. Previous studies have reported that certain lncRNAs, including Small nuclear RNA host gene 20 (SNHG20) (29), terminal differentiation-induced ncRNA (30), nuclear paraspeckle assembly transcript 1 (NEAT1) (31) and XIST (15) are aberrantly expressed in GC and regulate tumour 
A

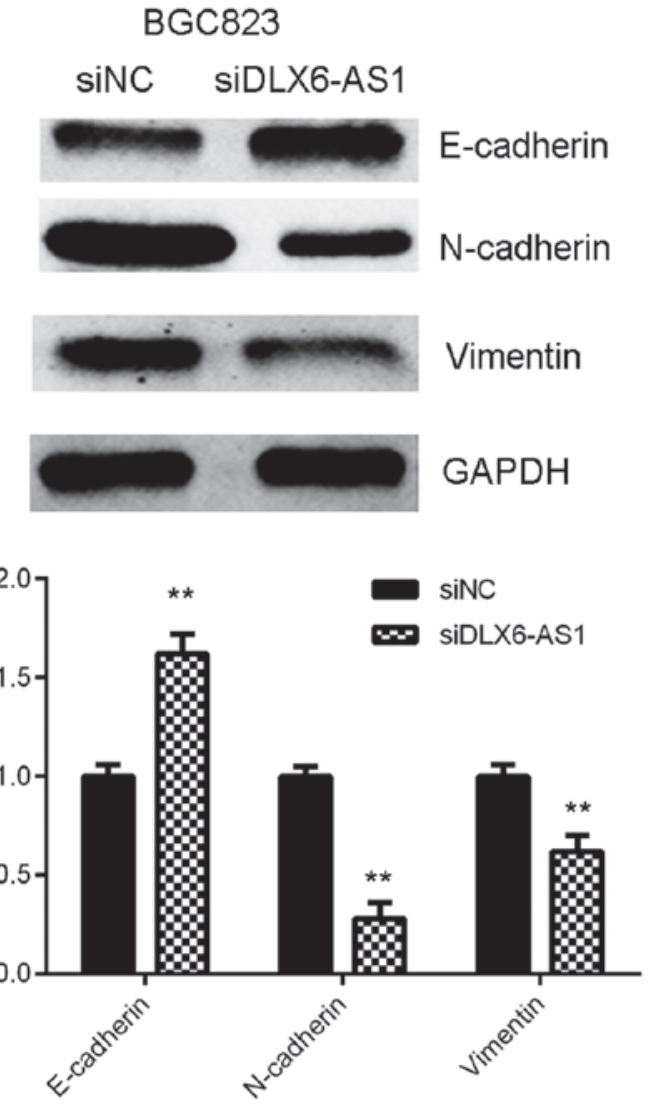

B

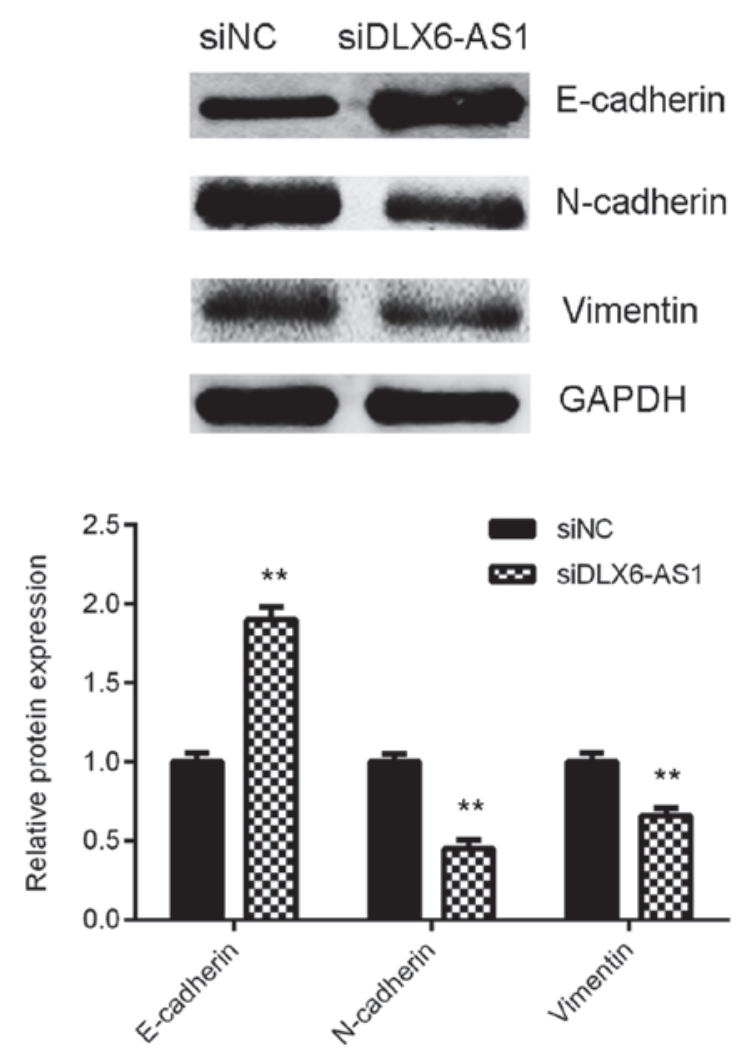

Figure 4. Silencing DLX6-AS1 inhibited epithelial-mesenchymal transition in gastric cancer cells. (A) BGC823 and (B) AGS cells were transfected with DLX6-AS1 siRNA or NC siRNA and western blotting was performed to determine E-cadherin, N-cadherin and Vimentin protein levels. GAPDH was used as internal reference. ${ }^{* *} \mathrm{P}<0.01$ vs. siNC. DLX6-AS1, distal-less homeobox 6 antisense 1; siRNA, small interfering RNA; NC, negative control.

growth and metastasis. For instance, NEAT1 is an unfavourable prognostic factor in GC and serves a promoting role in cell migration and invasion (31). SNHG20 promotes GC progression via the downregulation of p21 expression and the upregulation of the glycogen synthase kinase $3 \beta / \beta$-catenin signalling pathway (29). The results of the present study indicated that DLX6-AS1 is significantly upregulated in GC tissues and cell lines, compared with adjacent normal tissues and non-tumor GES-1 cells. In addition, it was revealed that the expression of DLX6-AS1 was higher in advanced GC tissue samples (III/IV) compared with early-stage samples (I/II). It was also demonstrated that a high DLX6-AS1 expression was significantly associated with lymph node metastasis and advanced TNM stage. Patients with GC and a high DLX6-AS1 expression exhibited decreased survival times compared with those with a low DLX6-AS1 expression. These results indicate that the upregulation of DLX6-AS1 may serve a key role in $\mathrm{GC}$ progression.

To further assess the function of DLX6-AS1 in GC growth and metastasis, loss-of-function assays were performed using AGS and BGC823 cells by transfecting DLX6-AS1-specific siRNA. It was demonstrated that silencing DLX6-AS1 expression caused a significant reduction in GC cell proliferation, colony formation, cell cycle progression, migration and invasion. Similarly, Zeng et al (18) revealed that DLX6-AS1 expression was also upregulated in renal cell carcinoma cells, which was significantly associated with tumour progression. In addition, Zhang et al (19) demonstrated that DLX6-AS1 promotes the proliferation, invasion and migration of hepatocellular carcinoma cells in vitro, as well as tumor growth in vivo. EMT, characterized by the loss of an epithelial phenotype and the acquisition of mesenchymal properties, serves a central role in cell migration and invasion and is closely associated with tumor metastasis (32). Many lncRNAs have been suggested to regulate EMT in various cancers (33-35). For instance, the overexpression of IncRNA colorectal neoplasia differentially expressed facilitates EMT and is associated with a poor prognosis of patients with intrahepatic cholangiocarcinoma (33). However, to the best of our knowledge, the detailed role of DLX6-AS1 in EMT regulation in GC cells has not previously been studied. The results of the present study revealed that the knockdown of DLX6-AS1 significantly promotes E-cadherin protein expression and suppresses $\mathrm{N}$-cadherin and vimentin protein expression in GC cells, indicating that EMT was inhibited. It is therefore reasonable to suggest that the suppressive effects of DLX6-AS1 downregulation in $\mathrm{GC}$ cell invasion and migration may occur via the inhibition of EMT.

In summary, the present study demonstrated that the lncRNA, DLX6-AS1, is upregulated in GC and serves an oncogenic role, indicating that DLX6-AS1 may serve as a novel therapeutic target for GC treatment.

\section{Acknowledgements}

Not applicable. 


\section{Funding}

No funding was received.

\section{Availability of data and materials}

All datasets used and/or generated during the present study are available from the corresponding author on reasonable request.

\section{Authors' contributions}

XF and YT collected clinical tissues, designed the study and wrote the manuscript. XF, WG, SW and WK performed the clinical and cell experiments.

\section{Ethics approval and consent to participate}

The current study was approved by the Ethics Committee of Xiangya Hospital (Changsha, China). All written informed consents have been obtained.

\section{Patient consent for publication}

All participants provided written informed consent for publication.

\section{Competing interests}

The authors declare that they have no competing interests.

\section{References}

1. Siegel RL, Miller KD and Jemal A: Cancer statistics, 2015. CA Cancer J Clin 65: 5-29, 2015.

2. Torre LA, Bray F, Siegel RL, Ferlay J, Lortet-Tieulent J and Jemal A: Global cancer statistics, 2012. CA Cancer J Clin 65: 87-108, 2015.

3. Röcken C: Molecular classification of gastric cancer. Expert Rev Mol Diagn 17: 293-301, 2017.

4. Silva A, Bullock M and Calin G: The clinical relevance of long non-coding RNAs in cancer. Cancers (Basel) 7: 2169-2182, 2015

5. Hung T and Chang HY: Long noncoding RNA in genome regulation: Prospects and mechanisms. RNA Biol 7: 582-585, 2010.

6. Wapinski $\mathrm{O}$ and Chang HY: Long noncoding RNAs and human disease. Trends Cell Biol 21: 354-361, 2011.

7. Clark MB and Mattick JS: Long noncoding RNAs in cell biology. Semin Cell Dev Biol 22: 366-376, 2011.

8. Xiao Z, Qu Z, Chen Z, Fang Z, Zhou K, Huang Z, Guo X and Zhang Y: LncRNA HOTAIR is a prognostic biomarker for the proliferation and chemoresistance of colorectal cancer via MiR-203a-3p-mediated Wnt/ß-catenin signaling pathway. Cell Physiol Biochem 46: 1275-1285, 2018.

9. Liu K, YaoH,Wen Y,ZhaoH,Zhou N,LeiS and Xiong L:Functional role of a long non-coding RNA LIFR-AS1/miR-29a/TNFAIP3 axis in colorectal cancer resistance to pohotodynamic therapy. Biochim Biophys Acta Mol Basis Dis 1864: 2871-2880, 2018

10. Li PF, Chen SC, Xia T, Jiang XM, Shao YF, Xiao BX and Guo JM: Non-coding RNAs and gastric cancer. World J Gastroenterol 20: 5411-5419, 2014

11. Gibb EA, Brown CJ and Lam WL: The functional role of long non-coding RNA in human carcinomas. Mol Cancer 10: 38, 2011.

12. Xu R, Zhu X, Chen F, Huang C, Ai K, Wu H, Zhang L and Zhao X LncRNA XIST/miR-200c regulates the stemness properties and tumourigenicity of human bladder cancer stem cell-like cells. Cancer Cell Int 18: 41, 2018.

13. Xiong W, Huang C, Deng H, Jian C, Zen C, Ye K, Zhong Z, Zhao X and Zhu L: Oncogenic non-coding RNA NEAT1 promotes the prostate cancer cell growth through the SRC3/IGF1R/AKT pathway. Int J Biochem Cell Biol 94: 125-132, 2018.
14. Peng Z, Liu C and Wu M: New insights into long noncoding RNAs and their roles in glioma. Mol Cancer 17: 61, 2018.

15. Zhang Q, Chen B, Liu P and Yang J: XIST promotes gastric cancer (GC) progression through TGF-betal via targeting miR-185. J Cell Biochem 119: 2787-2796, 2018.

16. Zhang $\mathrm{H}$ and Lu W: LncRNA SNHG12 regulates gastric cancer progression by acting as a molecular sponge of miR320. Mol Med Rep 17: 2743-2749, 2018.

17. Li J, Li P, Zhao W, Yang R, Chen S, Bai Y, Dun S, Chen X, Du Y, Wang Y, et al: Expression of long non-coding RNA DLX6-AS1 in lung adenocarcinoma. Cancer Cell Int 15: 48, 2015.

18. Zeng X, Hu Z, Ke X, Tang H, Wu B, Wei X and Liu Z: Long noncoding RNA DLX6-AS1 promotes renal cell carcinoma progression via miR-26a/PTEN axis. Cell Cycle 16: 2212-2219, 2017.

19. Zhang L, He X, Jin T, Gang L and Jin Z: Long non-coding RNA DLX6-AS1 aggravates hepatocellular carcinoma carcinogenesis by modulating miR-203a/MMP-2 pathway. Biomed Pharmacother 96: 884-891, 2017.

20. Livak KJ and Schmittgen TD: Analysis of relative gene expression data using real-time quantitative PCR and the 2(-Delta Delta C(T)) method. Methods 25: 402-408, 2001.

21. Lim S and Kaldis P: Cdks, cyclins and CKIs: Roles beyond cell cycle regulation. Development 140: 3079-3093, 2013.

22. Mowers EE, Sharifi MN and Macleod KF: Functions of autophagy in the tumor microenvironment and cancer metastasis. FEBS J 285: 1751-1766, 2018.

23. Luo J, Chen J, Li H, Yang Y, Yun H, Yang S and Mao X: LncRNA UCA1 promotes the invasion and EMT of bladder cancer cells by regulating the miR-143/HMGB1 pathway. Oncol Lett 14: 5556-5562, 2017.

24. Zhu H, Zheng T, Yu J, Zhou L and Wang L: LncRNA XIST accelerates cervical cancer progression via upregulating Fus through competitively binding with miR-200a. Biomed Pharmacother 105: 789-797, 2018.

25. Zhou Y, Chen Y, Ding W, Hua Z, Wang L, Zhu Y, Qian H and Dai T: LncRNA UCA1 impacts cell proliferation, invasion, and migration of pancreatic cancer through regulating miR-96/FOXO3. IUBMB Life 70: 276-290, 2018.

26. Kahroba H, Hejazi MS and Samadi N: Exosomes: From carcinogenesis and metastasis to diagnosis and treatment of gastric cancer. Cell Mol Life Sci Feb 8, 2019. Doi: 10.1007/s00018-019-03035-2.

27. Tan HY, Wang C, Liu G and Zhou X: Long noncoding RNA NEAT1-modualted miR-506 regulates gastric cancer development through targeting STAT3. J Cell Biochem 120: 4827-4836, 2018.

28. Ren K, Liu QQ, An ZF, Zhang DP and Chen XH: MiR-144 functions as tumor suppressor by targeting PIM1 in gastric cancer. Eur Rev Med Pharmacol Sci 21: 3028-3037, 2017.

29. Liu J, Liu L, Wan JX and Song Y: Long noncoding RNA SNHG20 promotes gastric cancer progression by inhibiting p21 expression and regulating the GSK-3beta/beta-catenin signaling pathway. Oncotarget 8: 80700-80708, 2017.

30. Chen Z, Liu H, Yang H, Gao Y, Zhang G and Hu J: The long noncoding RNA, TINCR, functions as a competing endogenous RNA to regulate PDK1 expression by sponging miR-375 in gastric cancer. Onco Targets Ther 10: 3353-3362, 2017.

31. Fu JW, Kong Y and Sun X: Long noncoding RNA NEAT1 is an unfavorable prognostic factor and regulates migration and invasion in gastric cancer. J Cancer Res Clin Oncol 142: 1571-1579, 2016.

32. Ye Y, Xiao Y, Wang W, Yearsley K, Gao JX, Shetuni B and Barsky SH: ERalpha signaling through slug regulates E-cadherin and EMT. Oncogene 29: 1451-1462, 2010.

33. Xia XL, Xue D, Xiang TH, Xu HY, Song DK, Cheng PG and Wang JQ: Overexpression of long non-coding RNA CRNDE facilitates epithelial-mesenchymal transition and correlates with poor prognosis in intrahepatic cholangiocarcinoma. Oncol Lett 15: 4105-4112, 2018.

34. Li C, Wan L, Liu Z, Xu G, Wang S, Su Z, Zhang Y, Zhang C, Liu X, Lei Z and Zhang HT: Long non-coding RNA XIST promotes TGF-beta-induced epithelial-mesenchymal transition by regulating miR-367/141-ZEB2 axis in non-small-cell lung cancer. Cancer Lett 418: 185-195, 2018.

35. Jia L, Tian Y, Chen Y and Zhang G: The silencing of LncRNA-H19 decreases chemoresistance of human glioma cells to temozolomide by suppressing epithelial-mesenchymal transition via the Wnt/beta-Catenin pathway. Onco Targets Ther 11: 313-321, 2018. 